\title{
On Algebraic Curves over Real Closed Fields. I
}

\author{
Manfred Knebusch \\ Institute for Advanced Study, Princeton, New Jersey 08540, USA
}

\section{§1. Introduction}

In this paper we are concerned with the absolute theory of a smooth irreducible complete algebraic curve $X$ defined over a real closed field $K$. Our theory is purely algebraic. It may be of interest even in the case that $K$ is the field $\mathbb{R}$ of real numbers, since as far as I know there does not exist a purely algebraic theory of curves over $\mathbb{R}$ in the literature.

Let $F$ denote the field $K(X)$ of rational functions on $X$ defined over our real closed field $K$. Clearly $F$ is a finitely generated field extension of $K$ of transcendency degree 1 . We regard $X$ as a scheme of dimension 1 over $K$. Thus the local rings $\mathcal{O}_{\mathfrak{p}}=\mathcal{O}_{X, \mathfrak{p}}$ of the closed points $\mathfrak{p}$ of $X$ are precisely all discrete valuation rings $A$ with $K \subset A \subset F$ and $F$ the quotient field of $A$. The intersection of all $\mathcal{O}_{p}$ is assumed to be $K$.

We never make explicit reference to the generic point of $X$. Thus from now on we use the word "point" instead of "closed point," and we denote the set of closed points of our scheme again by $X$. The residue class field $K(\mathfrak{p})=\mathcal{O}_{\mathfrak{p}} / \mathfrak{m}_{\mathfrak{p}}$ of a point $\mathfrak{p}$ of $X$ is isomorphic over $K$ either to $K$ itself or to the algebraic closure $K(\sqrt{-1})$ of $K$. We call the points of the first type the real points of $X$, and those of the second type the complex points of $X$. We denote the real points by capital roman letters $P, Q, R, \ldots$ and the set of all real points by $\gamma$. The set $\gamma$ is not empty if and only if the field $F$ is formally real, and in this case $\gamma$ contains infinitely many points. This is clear from E. Artin's work on positive definite functions, cf. [A] and $[\mathrm{Lg}]$.

Every $f$ in $F$ induces a map from $\gamma$ to $K \cup \infty$ assigning to a point $P$ with $f \in \mathcal{O}_{P}$ the image $f(P)$ of $f$ in $K(P)=K$ and to a point $P$ with $f$ not in $\mathcal{O}_{P}$ the value $\infty$. If $\gamma$ is not empty then $f$ is uniquely determined by this map from $\gamma$ to $K \cup \infty$, and this map will be again denoted by $f$. These functions $f: \gamma \rightarrow K \cup \infty$ will be called the real functions on $\gamma$.

Present address: Fachbereich Mathematik der Universität, Universitätsstr. 31, D-8400 Regensburg, Bundesrepublik Deutschland 
Assume now that $K$ is the field $\mathbb{R}$ of real numbers and $\gamma$ is not empty. Then $\gamma$ is a compact $C^{\infty}$-manifold of dimension 1 , hence a disjoint union $\gamma_{1} \cup \cdots \cup \gamma_{r}$ of manifolds $\gamma_{i}$ diffeomorphic to the circle $S^{1}$, cf. [M, p. $\left.55 \mathrm{ff}\right]$. In 1933 Witt proved the following three theorems about the real functions on $\gamma$ [W]:

I. If a real function $f$ is positive definite on $\gamma$, i.e. $f(P) \geqq 0$ or $f(P)=\infty$ for all $P$ in $\gamma^{1}$, then $f=g^{2}+h^{2}$ with $g, h$ in $F$.

II. Given a sign $\varepsilon_{i}= \pm 1$ for each component $\gamma_{i}, 1 \leqq i \leqq r$, there exists a real function $f \neq 0$ such that $\varepsilon_{i} f$ is positive definite on $\gamma_{i}$ simultaneously for $1 \leqq i \leqq r$.

III. Given on each $\gamma_{i}$ a finite even number of points there exists a real function $f \neq 0$ which changes sign precisely at these points.

Theorem I has been generalized to the case that $K$ is an arbitrary real closed field: By Artin's theory ([A, Lg]) every positive definite real function is a sum of squares, and as Pfister has shown in [P] every sum of squares in $F$ is already a sum of two squares. Similar statements hold true for function fields over $K$ of transcendency degree $>1$, cf. $[\mathrm{A}, \mathrm{Lg}, \mathrm{P}]$, and $\S 4$ of this paper. The proofs are purely algebraic. They do not use Tarski's principle to transfer "elementary statements" from $\mathbb{R}$ to other real closed fields.

The first part of the present paper is mainly devoted to the problem of proving theorems analogous to Witt's Theorems II and III over arbitrary real closed fields. Before attacking this problem we have to introduce a topology on $\gamma$ which generalizes the topology of $\gamma$ as a $C^{\infty}$-manifold in the case $K=\mathbb{R}$.

This is done as follows. We consider first the case that $X$ is the projective line $\mathbb{P}_{\mathbf{K}}^{1}$. Then $\gamma$ is the set $K \cup \infty$ which occurred already above. We introduce open intervals ] $a, b$ [ on $\gamma$ in the usual way: If $a$ and $b$ are in $K$ and $a<b$ then ] $a, b$ [ is defined as the set of all $x$ in $K$ with $a<x<b$. \{Recall that $K$ has a unique ordering.\} If $a, b \in K$ and $a>b$ then ] $a, b$ [ consists of the $x$ in $K$ with $x>a$ or $x<b$ and the point $\infty$. Finally for $a$ in $K$ the interval $] a, \infty$ [ is the set of all $x$ in $K$ with $x>a$, and $] \infty, a[$ is the set of all $x$ in $K$ with $x<a$.

We equip $K \cup \infty$ with the "interval topology." By definition this topology has the open intervals as a subbasis. Then for $X$ an arbitrary smooth irreducible complete curve over $K$ we introduce on $\gamma$ the coarsest topology such that all real functions $f: \gamma \rightarrow K \cup \infty$ are continuous. Every open interval of $K \cup \infty$ can be transformed by an automorphism of $\mathbb{P}_{K}^{1}$ into the interval ]0, $\infty$ [. Thus a subbasis of our topology on $\gamma$ is given by the sets $f^{-1}(] 0, \infty[)$ with $f$ running through $F^{*}:=F \backslash\{0\}$. The real functions on $\gamma$ separate points, as follows for example from the weak approximation theorem for valuations. Thus our topology on $\gamma$ is Hausdorff.

Notice that in the case $X=\mathbb{P}_{K}^{1}$ we obtain in this way again the interval topology. This is easily verified using the fact that for $X=\mathbb{P}_{K}^{1}$ the group $F^{*}$ is generated by $K^{*}$, the normed linear polynomials, and the normed definite quadratic polynomials in one variable over $K$.

To prove Witt's Theorems II and III over arbitrary real closed fields we now have to find the right definition of the "components" $\gamma_{1}, \ldots, \gamma_{r}$ of $\gamma$. These should be closed and open subsets of $\gamma$. In the case $K=\mathbb{R}$ the $\gamma_{i}$ are just the connected components of the topological space $\gamma$. But if $K$ is not isomorphic to $\mathbb{R}$ the space

1 We later shall use a slightly different definition of positive definite functions, cf. $\S 2$ 
$\gamma$ will be totally disconnected, and we have to look for a more sophisticated definition of the components $\gamma_{i}$. This definition will be given in $\S 2$. Then we shall be able to prove Theorem II immediately. At the end of $\S 2$ we obtain a rather intuitive description of the components $\gamma_{i}$ : Two points $P$ and $Q$ of $\gamma$ lie in the same component if and only if $f(P) f(Q)>0$ for every real function $f$ which has no zeros and no poles on $\gamma$.

In $\S 3$ we show that for a real function the number of points of odd order on a fixed component $\gamma_{i}$ is always even. In $\S 4$ we finally prove Theorem III for $K$ an arbitrary real closed field, replacing in this theorem the phrase "which changes sign precisely at these points" by "which has odd order precisely at these points." This is necessary since the geometry needed to justify the former phrase is not yet established in $\S 4$.

We never apply Tarski's principle. As far as I see there is no simple procedure at hand to obtain our results from the existing transcendental theory of real curves by use of this principle. On the other hand, our approach in this paper is not more difficult for $K$ an arbitrary real closed field than for $K=\mathbb{R}$, as it should be for a truly algebraic theory. Thus our theory would not be simplified by first proving the statements for $K=\mathbb{R}$ and then transferring them to other real closed fields.

The main tool used in our proofs of Theorems II and III are: i) Tsen's theorem, that the Brauer group of $F(\sqrt{-1})$ is trivial; ii) a residue theorem for differential valued quadratic forms over curves, developed in [GHKS]; iii) a theorem of Geyer [G], which states that the Herbrand quotient of the Galois cohomology of the Jacobian of $X$ is trivial. Geyer proves in [G] more generally a duality theorem for the Galois cohomology of an arbitrary abelian variety over a real closed field analogous to Tate's duality theorem [T] over $\mathfrak{p}$-adic fields.

The close connection between the Galois cohomology of the Jacobian and Theorems I-III had been made explicit by Geyer for $K=\mathbb{R}$ already in 1964 in a paper $\left[G_{1}\right]$, in which he proved these theorems in a much more algebraic way than Witt. Geyer's paper $\left[G_{1}\right]$ is an essential source for our present work. The papers $[\mathrm{G}]$ and $\left[\mathrm{G}_{1}\right]$ together also contain a proof of Theorem I over arbitrary real closed fields very different from the proof indicated above.

In $\S 5$ we develop the notion of an "orientation" of $\gamma$. Fixing an orientation on $\gamma$ we then define in $\S 6$ for two points $P \neq Q$ on a component of $\gamma$ the "open interval" ]$P, Q[$, and verify many of the pleasant properties of intervals we are accustomed to on the circle $S^{1}$.

In part II of the paper we shall continue our study of intervals. We then shall prove for a real function without poles on a given closed interval analogs of some standard theorems in analysis of one real variable: Intermediate value theorem, Rolle's theorem, existence of maxima and minima, criterion for local extrema.

The final sections of the paper will deal with quadratic forms. We shall determine the Witt ring $W(Z)$ of an arbitrary Zariski open part $Z$ of $X$, that means of an arbitrary irreducible smooth curve over $K$, and the Witt ring $W(F)$ of the function field $F$. Since our paper is based to a large extent on quadratic form techniques, these investigations have a natural place in it. The author believes that quadratic form techniques also are quite useful for an understanding of 
algebraic varieties of dimension $>1$ over real closed fields or even the field $\mathbb{R}$ of real numbers.

Acknowledgements. This paper has been written while the author visited the Institute for Advanced Study at Princeton. I thank both the I.A.S. and my colleagues at Regensburg for making this visit and this paper possible. I also gratefully acknowledge a travel grant from the Fulbright Foundation and partial support by a National Science Foundation grant. Finally, I thank Professor John Milnor for some helpful remarks on an earlier version of this paper.

\section{§ 2. Components and Definite Functions}

We first recall some notions from the theory of quadratic forms tailored to the needs of this paper. For $L$ a field of characteristic $\neq 2$ we denote by $W(L)$ the Witt ring of $L$. The elements of $W(L)$ are by definition the equivalence classes $[\varphi]$ of non-singular symmetric bilinear forms $\varphi$ over $L$. Here two forms $\varphi$ and $\psi$ over $L$ are called equivalent, $\varphi \sim \psi$, if

$$
\varphi \perp r \times\langle 1,-1\rangle \cong \psi \perp s \times\langle 1,-1\rangle
$$

with $r$ and $s$ integers $\geqq 0$, cf. [L] or [MH]. $\{r \times\langle 1,-1\rangle$ denotes the orthogonal sum of $r$ copies of the binary hyperbolic form. $\}$ Addition and multiplication on $W(L)$ are given by the orthogonal sum and the tensor product of forms. By $Q(L)$ we denote the group $L^{*} / L^{* 2}$ of square classes. We identify a square class $a L^{* 2}$ with the equivalence class $[\langle a\rangle]$ of the one-dimensional form $\langle a\rangle$, and we usually write again $\langle a\rangle$ for this class. Thus $Q(L)$ is a subgroup of the group of units of the ring $W(L)$.

Let $P$ be a real point of our irreducible complete smooth curve $X$ over the real closed field $K$, and let $t$ be a uniformizing parameteriof $X$ at $P$, i.e. a generator of the maximal ideal $m_{P}$ of the local ring $\mathcal{O}_{P}=\mathcal{O}_{X, P}$. We introduce mappings $\tau_{P}$ and $\partial_{P}$ from the Witt ring $W(F)$ of the function field $F$ of $X$ to the ring $\mathbb{Z}$ of integers in the following way: Given a form ${ }^{2} \varphi$ over $F$ we choose a diagonalization

$$
\varphi \cong\left\langle f_{1}, \ldots, f_{r}, t f_{r+1}, \ldots, t f_{n}\right\rangle
$$

with all $f_{i}$ units of $\mathcal{O}_{P}$ and $0 \leqq r \leqq n$. This is always possible. Then we put

$$
\begin{aligned}
& \tau_{P}(\varphi)=\sum_{i=1}^{r} \operatorname{sign} f_{i}(P), \\
& \partial_{P}(\varphi)=\sum_{i=r+1}^{n} \operatorname{sign} f_{i}(P) .^{3}
\end{aligned}
$$

$\left\{\operatorname{Read} \tau_{P}(\varphi)=0\right.$ if $r=0$ and $\partial_{P}(\varphi)=0$ if $r=n$. It can be shown that these integers do not depend on the choice of the diagonalization above (cf. [MH, p. 85] and recall that $W(K) \cong \mathbb{Z})$. It is now evident that $\tau_{P}(\varphi)$ and $\partial_{P}(\varphi)$ only depend on the equivalence class $[\varphi]$, and that these maps $\tau_{P}$ and $\partial_{P}$ from $W(F)$ to $\mathbb{Z}$ are additive. $\tau_{P}$ does not depend on the choice of $t$ but $\partial_{P}$ does.

\footnotetext{
"Form" always means "non-singular symmetric bilinear form"

${ }^{3} \operatorname{Sign}(a)=+1$ if $a>0$, and $\operatorname{sign}(a)=-1$ if $a<0$
} 
For $\langle f\rangle$ a square class we write $\tau_{P}(f), \partial_{P}(f)$ instead of $\tau_{P}(\langle f\rangle), \partial_{P}(\langle f\rangle)$. If the order ord $f$ of $f$ at $P$ is even then $\tau_{P}(f)= \pm 1$ and $\partial_{P}(f)=0$. Otherwise $\tau_{P}(f)=0$ and $\partial_{P}(f)= \pm 1$.

(2.2) Definition. We call a real function $f \neq 0$ definite at $P$ if $f$ has even order at $P$, and we then call $\tau_{P}(f)$ the sign of $f$ at $P$. We say that $f$ is positive definite at $P$ if $\tau_{P}(f)=+1$, and that $f$ is negative definite at $P$ if $\tau_{P}(f)=-1$. Thus $f$ is positive definite at $P$ if and only if $f$ has even order at $P$ and the following holds true: Choosing a real function $g \neq 0$ such that $h:=f g^{2}$ has no zero or pole at $P$, the function $h$ has value $h(P)>0$. For $\alpha$ a subset of $\gamma$ we call $f$ definite (positive def., negative def.) on $\alpha$ if $f$ is definite (positive def., negative def.) at every point of $\alpha$.

We now fix some Zariski open subset $Z$ of $X$, i.e. we omit from $X$ a finite set of points. Recall that we obtain in this way all irreducible smooth curves over $K$. We denote by $\beta$ the set of real points of $Z$, which is an open subset of $\gamma$. We denote by $Q(Z)$ the group of all square classes $\langle f\rangle$ in $Q(F)$ with $f$ of even order at all points $\mathfrak{p}$ in $Z$, and by $Q^{\prime}(Z)$ the group of all square classes $\langle f\rangle$ in $Q(F)$ with $f$ of even order at all points $P$ in $\beta$, i.e. $f$ definite on $\beta$. We finally denote by $Q^{+}(F)$ the group of square classes $\langle f\rangle$ with $f$ positive definite on $\gamma$. This group certainly contains all classes $\langle f\rangle$ with $f$ a sum of squares in $F$. Indeed, if $f \neq 0$ is a sum of $r$ squares, and $P$ is a point of $\gamma$, then we can write

$$
f=g^{-2}\left(h_{1}^{2}+\cdots+h_{r}^{2}\right)
$$

with $g$ in $F^{*}$, all $h_{i}$ in $\mathcal{O}_{P}$ and not all $h_{i}$ in $m_{P}$. Thus we obtain

$$
\tau_{P}(f)=\tau_{P}\left(f g^{2}\right)=+1 \text {. }
$$

(2.3) Theorem. Let $f \neq 0$ be a real function positive definite on $\beta$. Then $f$ is a sum of squares in $F$, and thus $f$ is positive definite on $\gamma$.

This theorem is due to Artin [A]. His proof roughly runs as follows: If $f$ would not be a sum of squares then there would exist an ordering $<$ of the field $F$ with $f<0$. But then there would exist infinitely many points $P$ on $\gamma$ with $f(P) \neq \infty$, $f(P)<0$ (cf. also [Lg, p. 387], and for a proof using quadratic form techniques $\left.\left[\mathrm{K}_{1}, \S 6\right]\right)$. Since $\gamma \backslash \beta$ is finite this is a contradiction.

Proposition. $Q^{\prime}(Z)=Q(Z) Q^{+}(F)$.

Before proving this proposition we have to introduce some more notations. We denote by $\bar{X}$ the curve $X \otimes_{K} \bar{K}$ over $\bar{K}:=K(\sqrt{-1})$ obtained from $X$ by base extensions from $K$ to $\bar{K}$, and by $E$ the function field $F(\sqrt{-1})$ of $\bar{X}$. Notice that $\bar{X}$ is again irreducible, smooth, and complete. We denote by $\operatorname{Div}(X)$ the group of divisors on $X$ and by $\operatorname{Div}(\bar{X})$ the group of divisors on $\bar{X}$, the composition in both groups written multiplicatively. As usual we regard $\operatorname{Div}(X)$ as a subgroup of $\operatorname{Div}(\bar{X})$. Let $\rho$ denote the involution $\neq$ id. of $\bar{K} / K$. The Galois group $G=\{1, \rho\}$ of $\bar{K} / K$ operates on $\bar{X}$, on $\operatorname{Div}(\bar{X})$, and on $E$. The action of $\rho$ on an element of one of these sets and of related sets occurring later will be denoted by a bar. Over a real point $P$ of $X$ there lies a unique point of $\bar{X}$, again denoted by $P$, but over a complex point $\mathfrak{p}$ there lie two different conjugate points $\mathfrak{q}, \overline{\mathfrak{q}}$ of $\bar{X}$. Thus, since $\bar{X}$ is unramified over $X$, the prime divisor $P$ of $X$ is also a prime divisor of $\bar{X}$, but the 
prime divisor $\mathfrak{p}$ of $X$ is the norm $\mathrm{N}(\mathfrak{q})=\mathfrak{q} \overline{\mathfrak{q}}$ of the prime divisor $\mathfrak{q}$ of $\bar{X}$. These facts imply that $\operatorname{Div}(X)$ coincides with the subgroup $\operatorname{Div}(\bar{X})^{G}$ of fixed elements of $\operatorname{Div}(\bar{X})$.

Now the proof of Proposition 2.4 runs as follows. If $\beta$ is empty then also $\gamma$ is empty and we have $Q^{\prime}(Z)=Q(F)=Q^{+}(F)$. Assume from now on that $\beta$ is not empty. Let $f$ be a real function $\neq 0$ definite on $\beta$. Since $f$ has even order everywhere on $\beta$ the divisor of $f$ can be written as follows:

$$
\operatorname{div}(f)=\mathfrak{a}^{2} \mathrm{~N}(\mathfrak{b}) \mathfrak{c}
$$

with $\mathfrak{a}$ a divisor having support in $\beta, \mathfrak{c}$ a divisor with support in $\gamma \backslash \beta$ and $\mathrm{N}(\mathfrak{b})$ the norm $\mathfrak{b} \bar{b}$ of some divisor $\mathfrak{b}$ of $\bar{X}$. Changing $\mathfrak{a}$ and $\mathfrak{b}$ by suitable inverse powers of a prime divisor $P$ in $\beta$ we may assume that $b$ has degree zero. Now the group $J:=\operatorname{Pic}_{0}(\bar{X})$ of divisor classes of degree zero can be ragarded as an abelian variety over $\vec{K}$, the Jacobian of $\bar{X}$. Thus $J$ is a divisible group (e.g. [Mu, p. 42]). In particular there exists an equation

$$
\mathfrak{b}=\operatorname{div}(g) \mathfrak{v}^{2}
$$

with $g$ in $E^{*}$ and $\mathfrak{v}$ a divisor on $\bar{X}$. We then get

$$
\operatorname{div}(f)=\operatorname{div}(\mathrm{N}(g)) \cdot \mathrm{N}(\mathfrak{v})^{2} \cdot \mathfrak{a}^{2} \cdot \mathfrak{c}
$$

with $\mathrm{N}(g)$ denoting the norm $g \bar{g}$ of $g$. From this we learn that the real function $h:=f \mathrm{~N}(g)^{-1}$ has even order everywhere on $Z$. On the other hand $g=g_{1}+g_{2} \sqrt{-1}$ with elements $g_{i}$ of $F$, hence

$$
\mathrm{N}(g)=g_{1}^{2}+g_{2}^{2} \text {. }
$$

Thus we have

$$
\langle f\rangle=\langle h\rangle\langle\mathrm{N}(g)\rangle
$$

with $\langle h\rangle$ in $Q(Z)$ and $\langle\mathrm{N}(g)\rangle$ in $Q^{+}(F)$.

We now want to prove

(2.5) Proposition. $Q(Z)$ is a finite group.

Clearly for any point $\mathfrak{p}$ of $Z$ we have

$(Q(Z \backslash\{\mathfrak{p}\}): Q(Z)) \leqq 2$.

Thus since $X \backslash Z$ is finite also the index $(Q(Z): Q(X))$ is finite, and it suffices to show that $Q(X)$ is finite.

We regard the map $v$ from $Q(X)$ to the group $\operatorname{Pic}(X)$ of divisor classes on $X$ assigning to a square class $\langle f\rangle$ the class of the $\operatorname{divisor} \frac{1}{2} \operatorname{div}(f)$. This map is a well defined group homomorphism. Clearly the image of $v$ is the subgroup $\operatorname{Pic}(X)_{2}$ of elements of order $\leqq 2$ in $\operatorname{Pic}(X)$. The kernel of $v$ consists of the square classes of constant functions. Thus we obtain an exact sequence

$$
1 \rightarrow Q(K) \rightarrow Q(X) \stackrel{v}{\longrightarrow} \operatorname{Pic}(X)_{2} \rightarrow 1,
$$

the map from $Q(K)$ to $Q(X)$ being injective since $K$ is algebraically closed in $F$. \{In fact, an exact sequence analogous to (2.6) exists for an arbitrary complete scheme over a field, cf. [K, 2.3.1].\} 
$Q(K)$ consists of two elements $\langle \pm 1\rangle$, and we are left to show that $\operatorname{Pic}(X)_{2}$ is finite. For this we relate $\operatorname{Pic}(X)$ to $\operatorname{Pic}(\bar{X})$, using cohomology of the Galois group $G=\{1, \rho\}$. For $A$ a $G$-module we denote by $H^{0}(A)$ and $H^{1}(A)$ the cohomology groups

$$
H^{0}(A)=A_{1-\rho} /(1+\rho) A, \quad H^{1}(A)=A_{1+\rho} /(1-\rho) A
$$

with $A_{1 \pm \rho}$ the kernel of $1 \pm \rho$ on $A$. Consider first the canonical exact sequence of $G$-modules

$$
1 \rightarrow \bar{K}^{*} \rightarrow E^{*} \rightarrow \mathfrak{H}(\bar{X}) \rightarrow 1
$$

with $\mathfrak{S}(\bar{X})$ the group of principal divisors on $\bar{X}$. Regarding the $G$-invariant elements we obtain an exact sequence

$$
1 \rightarrow K^{*} \rightarrow F^{*} \rightarrow \mathfrak{H}(\bar{X})^{G} \rightarrow 1,
$$

since $H^{1}\left(\bar{K}^{*}\right)=0$. Thus the canonical map from $\mathfrak{H}(X)$ to $\mathfrak{H}(\bar{X})^{G}$ is bijective. Furthermore since $H^{1}\left(E^{*}\right)=0$ we have an exact sequence

$$
1 \rightarrow H^{1}(\mathfrak{H}(\bar{X})) \rightarrow H^{0}\left(\bar{K}^{*}\right) \rightarrow H^{0}\left(E^{*}\right) .
$$

Now $H^{0}\left(\bar{K}^{*}\right)=K^{*} / \mathrm{N}\left(\bar{K}^{*}\right)$ consists of two norm classes (= square classes) represented by \pm 1 . Since -1 is not a norm of $E / F$ the map from $H^{0}\left(\bar{K}^{*}\right)$ to $H^{0}\left(E^{*}\right)$ is injective, and we learn that

$$
H^{1}(\mathfrak{H}(\bar{X}))=0 .
$$

Regarding now the $G$-invariant elements of the canonical exact sequence

$$
1 \rightarrow \mathfrak{H}(\bar{X}) \rightarrow \operatorname{Div}(\bar{X}) \rightarrow \operatorname{Pic}(\bar{X}) \rightarrow 1
$$

we see that the sequence

$$
1 \rightarrow \mathfrak{H}(X) \rightarrow \operatorname{Div}(X) \rightarrow \operatorname{Pic}(\bar{X})^{\mathfrak{G}} \rightarrow 1
$$

is exact. Thus we have shown

(2.7) Lemma. The canonical map from $\operatorname{Pic}(X)$ to $\operatorname{Pic}(\bar{X})^{G}$ is bijective $(\gamma$ not empty).

By this lemma $\operatorname{Pic}(X)_{2}$ is the subgroup of $G$-invariant elements of $\operatorname{Pic}(\bar{X})_{2}$. Now the elements of order $\leqq 2$ of $\operatorname{Pic}(\bar{X})$ lie in the group $J$ of divisor classes of degree zero on $\bar{X}$. Since $J$ is an abelian variety over $\bar{K}$ of dimension $g$, the genus of $X$, the set $J_{2}$ of elements of order $\leqq 2$ in $J$ contains precisely $2^{28}$ elements [Mu, p. 64]. Putting all facts together we see that $Q(X)$ contains at most $2^{28+1}$ elements.

From the Propositions (2.4) and (2.5) now proved we immediately obtain

(2.8) Corollary. For $Z$ a Zariski open subset of $X$ the group $Q^{\prime}(Z) / Q^{+}(F)$ is finite.

Now we are able to define the components of the set $\beta$ of real points of $Z$. Assume $\beta$ is not empty. We call two points $P$ and $Q$ of $\beta$ equivalent if $\tau_{P}(f)=\tau_{Q}(f)$ for every real function $f \neq 0$ definite on $\beta$. According to our Corollary (2.8) the set $\beta$ decomposes into finitely many equivalence classes $\beta_{1}, \ldots, \beta_{t}$ with respect to this relation. These $\beta_{i}$ we call the components of $\beta$. 
(2.9) Proposition. Every component $\beta_{i}$ of $\beta$ is open and closed in $\beta$.

Proof. We fix some point $P$ in $\beta$ and denote by $\alpha$ the component containing $P$. Let $Q$ be a point in $\beta \backslash \alpha$. There exists a real function $f \neq 0$ definite on $\beta$ with $\tau_{P}(f)=-1$ and $\tau_{Q}(f)=+1$. The orders of $f$ at $P$ and $Q$ are even numbers $2 n$ and $2 m$. By the weak approximation theorem for valuations there exists a real function $g$ with orders $-n$ and $-m$ at $P$ and $Q$ respectively. The function $h:=f g^{2}$ is definite on $\beta$ and $h(P) \neq \infty, h(Q) \neq \infty, h(P)<0, h(Q)>0$. From this study we learn that $\beta \backslash \alpha$ is the union of the open sets $h^{-1}(] 0, \infty[)$ with $h$ running through all definite functions on $\beta$ with $h(P) \neq \infty$ and $h(P)<0$. Thus $\alpha$ is closed. Since $\beta \backslash \alpha$ is a union of components, also $\beta \backslash \alpha$ is closed, i.e. $\alpha$ is open. q.e.d.

(2.10) Theorem. Given a sign $\varepsilon_{i}= \pm 1$ for each component $\beta_{i}, 1 \leqq i \leqq t$, there exists a real function $f \neq 0$ such that $f$ is definite on each $\beta_{i}$ with sign $\varepsilon_{i}$.

To prove this theorem we need the following well-known

(2.11) Lemma. For $f$ and $g$ arbitrary elements of $F^{*}$ there exists an element $h$ in $F^{*}$ such that the quadratic forms $\langle 1,-f\rangle \otimes\langle 1,-g\rangle$ and $2 \times\langle 1,-h\rangle$ over $F$ are isomorphic.

Indeed, this statement means that the quaternion algebra $[f, g]$ over $F$ with structure coefficients $f, g$ is split by $F(\sqrt{-1})$. This follows from Tsen's theorem that over $F(\sqrt{-1})$ there do not exist non trivial central division algebras of finite rank.

The proof of Theorem (2.10) now runs as follows. We may assume $t \geqq 2$. It suffices to find a real function $f \neq 0$ definite on $\beta$ which has sign -1 on a fixed component, say $\beta_{1}$, and has sign +1 on all other components.

For every $i$ with $2 \leqq \mathrm{i} \leqq t$ there exists a function $g_{i} \neq 0$ definite on $\beta$ with sign -1 on $\beta_{1}$ and sign +1 on $\beta_{i}$. Applying Lemma (2.11) iteratively we obtain

$$
\left\langle 1,-g_{2}\right\rangle \otimes \cdots \otimes\left\langle 1,-g_{t}\right\rangle \cong 2^{t-2} \times\langle 1,-f\rangle
$$

with some $f$ in $F^{*}$. Let $P$ be an arbitrary point of $\beta$. Applying $\partial_{P}$ to this relation we see that $\partial_{P}(f)=0$. Thus $f$ is definite on $\beta$. Now $\tau_{P}$ is a ring homomorphism on the subring of $W(F)$ generated by $Q^{\prime}(Z)$. Thus applying $\tau_{P}$ to our relation we obtain

$$
2^{t-2}\left(1-\tau_{P}(f)\right)=\left(1-\tau_{P}\left(g_{2}\right)\right) \ldots\left(1-\tau_{P}\left(g_{t}\right)\right) \text {. }
$$

If $P$ lies in some $\beta_{i}$ with $i \geqq 2$ then the right hand side is zero, since $\tau_{P}\left(g_{i}\right)=+1$. But if $P$ lies in $\beta_{1}$ then the right hand side is $2^{t-1}$. Thus $\tau_{P}(f)=+1$ if $P$ lies in $\beta \backslash \beta_{1}$ and $\tau_{P}(f)=-1$ if $P$ lies in $\beta_{1}$.

Theorem (2.10), now proved, and the previous Theorem (2.3) together imply

(2.12) Corollary. The group $Q^{\prime}(Z) / Q^{+}(F)$ has order $2^{t}$ with $t$ the number of components of $\beta$.

We finally point out that sharper conditions can be imposed on the function $f$ in Theorem (2.10).

(2.13) Proposition. Let $\mathfrak{p}$ be a fixed complex point of $X$. Then the function $f$ in Theorem (2.10) can be chosen such that $f$ in addition is regular on $X \backslash\{\mathfrak{p}\}$ and has no zeros on $\beta$. 
This follows immediately from Theorem (2.10) and

(2.14) Lemma. Let $P_{1}, \ldots, P_{t}$ be finitely many different points on $\gamma$ and $n_{1}, \ldots, n_{t}$ be integral numbers. Further let $\mathfrak{p}$ be a complex point of $X$. Then there exists a real function $f \neq 0$ which is positive definite on $\gamma$ and has moreover the following properties:

a) $f$ has at $P_{i}$ the order $2 n_{i}, 1 \leqq i \leqq t$.

b) $f$ has no zeros on $\gamma \backslash\left\{P_{1}, \ldots, P_{t}\right\}$.

c) $f$ is regular at all points of $X$ different from $P_{1}, \ldots, P_{t}$, and $\mathfrak{p}$.

The proof of this Lemma runs as follows. There exists a function $g$ in $F^{*}$ which has order $n_{i}$ at $P_{i}$ for $1 \leqq i \leqq t$ and has a pole outside $\left\{P_{1}, \ldots, P_{t}\right\}$ at most at $\mathfrak{p}$. This is a well-known consequence of "Riemann's part" of the Riemann-Roch theorem. If $g$ has no zeros on $\gamma \backslash\left\{P_{1}, \ldots, P_{t}\right\}$ then we are through with $f:=g^{2}$. Assume now that $g$ has on $\gamma \backslash\left\{P_{1}, \ldots, P_{t}\right\}$ the zeros $Q_{1}, \ldots, Q_{s}$. There exists a function $h$ on $F^{*}$ with

a) $h$ has order $n_{i}$ at $P_{i}, 1 \leqq i \leqq t$;

b) $h$ has order 0 at $Q_{j}, 1 \leqq j \leqq s$;

c) $h$ is regular at all points of $X$ different from $P_{1}, \ldots, P_{t}$, and $\mathfrak{p}$.

The function $f:=g^{2}+h^{2}$ clearly has the properties stated in the lemma.

Using a part of Proposition (2.13), now proved, we obtain a description of the components of $\beta$ which is slightly more intuitive than the definition of the components above.

(2.15) Corollary. Two points $P$ and $Q$ of $\beta$ lie in the same component of $\beta$ if and only if $f(P) f(Q)>0$ for every real function $f$ which has no zeros or poles on $\beta$. We even may restrict $f$ to those functions which are regular on $X \backslash\{\mathfrak{p}\}$ for some fixed complex point $p$ and have no zeros on $\beta$.

\section{§3. A Residue Theorem}

Throughout the paper we denote the components of the space $\gamma$ of real points of $X$ by $\gamma_{1}, \ldots, \gamma_{r}\{r=0$ if $\gamma$ is empty $\}$. We now regard non-singular bilinear forms over $F$ with values in the vector space $\Omega_{F}=\Omega_{F / K}$ of rank one over $F$ consisting of the rational differentials $f d g$ on $X$. We have a Witt group $W\left(\Omega_{F}\right)$ of equivalence classes of these forms, defined in an obvious way, which is a free module of rank one over the ring $W(F)$ [GHKS]. Notice that any $F$-linear bijective map from $F$ onto $\Omega_{F}$ yields an isomorphism from $W(F)$ onto $W\left(\Omega_{F}\right)$, but that no canonical isomorphism from $W(F)$ onto $W\left(\Omega_{F}\right)$ is at hand.

For every real point $P$ of $X$ we have an additive "residue map"

$$
\text { res }_{P}: W\left(\Omega_{F}\right) \rightarrow \mathbb{Z}
$$

as follows (cf. (2.1)): Let $t$ be a uniformizing parameter of $X$ at $P$, and let $\varphi$ be a differential valued form over $F$. We choose a diagonalization

$$
\varphi \cong\left\langle f_{1} d t, \ldots, f_{r} d t, f_{r+1} t^{-1} d t, \ldots, f_{n} t^{-1} d t\right\rangle
$$


with real functions $f_{i}$ having no pole or zero at $P$ and $0 \leqq r \leqq n$, and we map the Witt class $[\varphi]$ to

$$
\operatorname{res}_{P}(\varphi):=\sum_{i=r+1}^{n} \operatorname{sign} f_{i}(P) .
$$

Notice that $\operatorname{res}_{P}(\varphi)$ does not depend on our choice of the uniformizing parameter $t$.

In [GHKS] a residue theorem for differential valued forms has been established for complete smooth curves over arbitrary fields of constants, which in our special case of a real closed constant field reads as follows: For $\varphi$ a differential valued form over $F$ the residues $\operatorname{res}_{P}(\varphi)$ vanish for almost all $P$ in $\gamma$ and

$$
\sum_{P \in \gamma} \operatorname{res}_{P}(\varphi)=0 .
$$

\{Residues can also be defined at the complex points of $X$ but these residues do not contribute anything to the sum formula.\} The sum formula (3.2) can be refined as follows:

(3.3) Theorem. For every component $\gamma_{i}, 1 \leqq i \leqq r$, and every differential valued form $\varphi$ over $F$

$$
\sum_{P \in \gamma_{i}} \operatorname{res}_{P}(\varphi)=0 .
$$

Proof. We may assume $r \geqq 2$. We prove the formula for, say, $i=1$. According to Theorem (2.10) there exists a real function $f \neq 0$ which is definite on $\gamma$ and has sign -1 on $\gamma_{1}$ but sign +1 on the other components. For $\mathrm{P}$ an arbitrary real point we have

$$
\operatorname{res}_{P}(\langle f\rangle \otimes \varphi)=\tau_{P}(f) \operatorname{res}_{P}(\varphi)
$$

since $f$ has even order at $P$. Thus $\operatorname{res}_{P}(\langle f\rangle \otimes \varphi)$ equals $\operatorname{res}_{P}(\varphi)$ for $P$ in $\gamma \backslash \gamma_{1}$ and $-\operatorname{res}_{P}(\varphi)$ for $P$ in $\gamma_{1}$. Writing down the formula (3.2) for $\varphi$ and for $\langle f\rangle \otimes \varphi$ yields the theorem. q.e.d.

As a first consequence of this theorem we have

(3.4) Theorem. For every real function $f \neq 0$ and every component $\gamma_{i}$ the number of $\gamma_{i}$ at which fhas odd order is even.

Proof. We choose a rational differential $\omega \neq 0$ whose divisor has a support disjoint from the divisor of $f$. Then we can roughly say the following about the residues of the one-dimensional form $\langle f \omega\rangle$ :

$$
\operatorname{res}_{P}\langle f \omega\rangle= \pm \operatorname{res}_{P}\langle\omega\rangle
$$

for $P$ in the support of $\operatorname{div}(\omega)$,

$$
\operatorname{res}_{P}\langle f \omega\rangle= \pm 1
$$

if $f$ has odd order in $P$, finally

$$
\operatorname{res}_{p}\langle f \omega\rangle=0
$$


for all other real points $P$. Thus we obtain mod 2

$$
\sum_{P \in \gamma_{i}} \operatorname{res}_{P}\langle f \omega\rangle \equiv \sum_{P \in \gamma_{i}} \operatorname{res}_{P}\langle\omega\rangle+\sum_{P \in \gamma_{i}} \operatorname{ord}_{P}(f) .
$$

Using the residue Theorem (3.3) we get

$$
\sum_{P \in \gamma_{i}} \operatorname{ord}_{P}(f) \equiv 0 \bmod 2 . \quad \text { q.e.d. }
$$

We can now establish Harnack's theorem over arbitrary real closed fields:

(3.5) Theorem. The number $r$ of components of $\gamma$ does not exceed $g+1$ with $g$ the genus of $X$.

Indeed, the proof of Harnack's theorem on the last page of Lang's book [ $\left.\mathrm{Lg}_{1}\right]$ uses beside the theorem of Riemann-Roch precisely the statement of the preceding theorem, and thus remains valid over an arbitrary real closed field of constants.

\section{§4. The Main Theorem}

We first quote

(4.1) Theorem. Every real function $f \neq 0$ which is positive definite on $\gamma$ is a sum of two squares in $F$.

Starting with Artin's Theorem 2.3 this theorem is a special case of Pfister's theorem [P], that for $A$ a field extension of $K$ of transcendency degree $n$ every sum of squares in $A$ is a sum of $2^{n}$ squares. From a present day view (1975) the most natural way to deduce Pfister's theorem from general principles is perhaps the following: ${ }^{4}$ Let $B=A(\sqrt{-1})$. As for any quadratic field extension we have an exact triangle [L, p. 201]

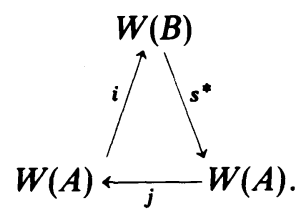

Here $i$ denotes the natural map from $W(A)$ to $W(B), j$ denotes multiplication by the discriminant form $\langle 1,1\rangle$ of $B / A$, and $s^{*}$ denotes the transfer map $[\varphi] \mapsto[s \circ \varphi]$ with $s$ the $A$-linear map from $B$ to $A$ defined by $s(1)=0, s(\sqrt{-1})=1$. We denote by $I(A)$ and $I(B)$ the fundamental ideals of the rings $W(A), W(B)$, i.e. the ideals consisting of the classes of forms of even dimension, and by $I^{m}(A), I^{m}(B)$ their $m$-th power $(m \geqq 0)$. Our exact triangle induces a complex

$$
\begin{aligned}
& 0 \rightarrow W(A) \stackrel{i}{\longrightarrow} W(B) \stackrel{s^{*}}{\longrightarrow} W(A) \stackrel{j}{\longrightarrow} I(A) \stackrel{i}{\longrightarrow} \\
& \stackrel{i}{\longrightarrow} I(B) \stackrel{s^{*}}{\longrightarrow} I(A) \stackrel{i}{\longrightarrow} I^{2}(A) \rightarrow \cdots
\end{aligned}
$$

4 I am indebted to J.K. Arason for this argument 
which at least is "exact on Pfister forms," cf. [Ar, § 2] or [EL, § 2]. Now let $w \neq 0$ be a sum of squares in $A$ and let $\varphi$ denote the Pfister form $2^{n-1} \times\langle 1,-w\rangle$. Assume $\varphi$ is not hyperbolic. Otherwise $w$ would be already a sum of $2^{n-1}$ squares. There exists a number $r \geqq 0$ such that $2^{r} \times \varphi$ is anisotropic but $2^{r+1} \times \varphi$ is hyperbolic. Since the class $\left[2^{r} \times \varphi\right]$ is mapped to zero under $j$ there exists a Pfister form $\psi$ of dimension $2^{r+n}$ over $B$ with $s^{*}(\psi) \sim 2^{r} \times \varphi$. If $r$ would be $\geqq 1$ then by a wellknown theorem of Tsen $\psi$ would be isotropic and hence hyperbolic, which contradicts the assumption that $2^{r} \times \varphi$ is not hyperbolic. Thus $r=0$, i.e. $2 \times \varphi \sim 0$. This means that $w$ is a sum of $2^{n}$ squares.

As already pointed out in $\S 1$, also Geyer's proof of Theorem (4.1) for $K=$ $\mathbb{R}\left[\mathrm{G}_{1}\right]$ generalizes to arbitrary real closed fields of constants. Indeed, the only non-trivial fact used by Geyer to prove this theorem is the statement that the Herbrand quotient of the Jacobian $J$ is always trivial, and this statement has recently been proved by Geyer over arbitrary real closed fields:

(4.2) Theorem [G]. Let $A$ be an abelian variety defined over a real closed field $K$, and identify $A$ with the $G$-module of its points rational over $\bar{K}$. The cohomology groups $H^{0}(A)$ and $H^{1}(A)$ are finite and have the same cardinality.

Theorem (4.2) plays a central role in our paper, since it leads to the Theorem III discussed in $\S 1$. This will be explained now.

Assuming from now on that $\gamma$ is not empty we give an interpretation of $H^{0}(J)$ and $H^{1}(J)$ in terms of the behavior of real functions on $\gamma$ following Geyer's paper $\left[\mathrm{G}_{1}\right]$. We use the following abbreviations:

$D=\operatorname{Group} \operatorname{Div}(\bar{X})$ of divisors on $\bar{X}$;

$D_{0}=$ Subgroup $\operatorname{Div}_{0}(\bar{X})$ of divisors of degree zero;

$\mathfrak{H}=$ Subgroup $\mathfrak{H}(\bar{X})$ of principal divisors on $\bar{X}$;

$J=D_{0} / \mathfrak{H}=$ group $\operatorname{Pic}_{0}(\bar{X})$ of divisor classes of degree zero (as above).

We observed already in $\S 2$ that we have natural isomorphisms

$\operatorname{Div}(X) \stackrel{\sim}{\longrightarrow} D^{G}, \quad$ hence $\operatorname{Div}_{0}(X) \stackrel{\sim}{\longrightarrow} D_{0}^{G}$;

$\mathfrak{H}(X) \stackrel{\sim}{\longrightarrow} \mathfrak{H}^{G} ; \quad \operatorname{Pic}(X) \stackrel{\sim}{\longrightarrow} \operatorname{Pic}(\bar{X})^{G}$,

hence $\operatorname{Pic}_{0}(X) \stackrel{\sim}{\longrightarrow} J^{G}$. We also have seen in $\S 2$ that $H^{1}(\mathfrak{H})=0$.

The trivial sequence

$1 \rightarrow D_{0} \rightarrow D \stackrel{\text { deg }}{\longrightarrow} \mathbb{Z} \rightarrow 0$

implies an exact sequence

$H^{0}(D) \rightarrow H^{0}(\mathbb{Z}) \rightarrow H^{1}\left(D_{0}\right) \rightarrow H^{1}(D)$.

Since $D$ is a direct sum of $G$-modules isomorphic to $\mathbb{Z}[G]$ or to $\mathbb{Z}$ with trivial action, we have $H^{1}(D)=0$. Furthermore $H^{0}(\mathbb{Z})=\mathbb{Z} / 2 \mathbb{Z}$. Since there exist prime divisors of degree 1 invariant under $G\left(\gamma\right.$ is not empty), the map from $H^{0}(D)$ to $H^{0}(\mathbb{Z})$ is surjective, and we learn that $H^{1}\left(D_{0}\right)=0$.

Considering now the trivial sequence

$1 \rightarrow \mathfrak{H} \rightarrow D_{0} \rightarrow J \rightarrow 1$ 
we obtain an exact sequence

$$
1 \rightarrow H^{1}(J) \rightarrow H^{0}(\mathfrak{H}) \rightarrow H^{0}\left(D_{0}\right) \rightarrow H^{0}(J) \rightarrow 1
$$

since $H^{1}\left(D_{0}\right)$ and $H^{1}(\mathfrak{S})$ are trivial. We clearly have a canonical isomorphism

$$
H^{1}(J) \cong\{\mathfrak{a} \in D \mid \mathfrak{a} \overline{\mathfrak{a}} \text { principal }\} /\left\{\mathfrak{b} \overline{\mathfrak{b}}^{-1} \mid \mathfrak{b} \in D\right\} \mathfrak{H} \text {. }
$$

Furthermore since $\mathfrak{H}^{G} \cong \mathfrak{H}(X)$ we have a canonical isomorphism

$$
H^{0}(\mathfrak{S}) \cong F^{*} / K^{*} \cdot \mathrm{N}_{E / F}\left(E^{*}\right) .
$$

Using Theorem (4.1) we may write

$$
H^{0}(\mathfrak{S}) \cong Q(F) /\{ \pm 1\} Q^{+}(F) .
$$

The map from $H^{1}(J)$ to $H^{0}(\mathfrak{S})$ sends a class [a] with $\mathfrak{a} \overline{\mathfrak{a}}=\operatorname{div}(f)$ to the class $[f]$ in $H^{0}(\mathfrak{S})$. The functions $f$ arising in this way are precisely all real functions $f \neq 0$ which have even order everywhere on $\gamma$. Thus the initial part of our exact sequence (4.3) yields a natural isomorphism

$$
H^{1}(J) \stackrel{\sim}{\longrightarrow} Q^{\prime}(X) /\{ \pm 1\} Q^{+}(F) .
$$

In particular $H^{1}(J)$ contains precisely $2^{r-1}$ elements according to Corollary (2.12). We now interpret $H^{0}(J)$. We identify

$$
H^{0}\left(D_{0}\right)=\operatorname{Div}_{0}(X) / \mathrm{N}\left(\operatorname{Div}_{0}(\bar{X})\right)
$$

in the obvious way with the group of formal products $\prod_{P \in \gamma} P^{\varepsilon_{P}}$ with exponents $\varepsilon_{P}$
in $\mathbb{Z} / 2 \mathbb{Z}$, almost all $\varepsilon_{P}=0$, and $\sum_{P \in \gamma} \varepsilon_{P}=0$.

From our exact sequence (4.3) we get

$$
H^{0}(J) \cong H^{0}\left(D_{0}\right) / \text { Image of } H^{0}(\mathfrak{S}) \text {. }
$$

Now by Theorem (3.4) the image of $H^{0}(\mathfrak{Y})$ is contained in the subgroup $H^{\prime}$ of $H^{0}\left(D_{0}\right)$ consisting of all products $\prod P^{\varepsilon_{P}}$ with

$$
\sum_{P \in y_{i}} \varepsilon_{P}=0
$$

for $1 \leqq i \leqq r$. But by Geyer's Theorem (4.2) we know that $H^{0}(J)$ has the same cardinality $2^{r-1}$ as $H^{1}(J)$. Thus the image of $H^{0}(\mathfrak{H})$ in $H^{0}\left(D_{0}\right)$ coincides with $H^{\prime}$. In other words:

(4.5) Main Theorem. Given on each $\gamma_{i}$ a finite even number of points, there exists a real function $f \neq 0$ which has odd order on $\gamma$ precisely at these points.

Applying the previous Lemma (2.14) we obtain a slightly stronger version of this theorem.

(4.5 a) Theorem. Given on each $\gamma_{i}$ a finite even number of points and given a complex point $\mathfrak{p}$ on $X$ there exists a real function $f \neq 0$, which is regular on $X \backslash\{\mathfrak{p}\}$, has a simple zero at each of the given points on the $\gamma_{i}$, and has no other zeros on $\gamma$. 


\section{§5. Orientations}

We continue to assume that $\gamma$ is not empty. Our first goal in this section is to develop a criterion that a given divisor class $[a]$ in $\operatorname{Pic}(X)$ lies in $2 \operatorname{Pic}(X)$.

Consider the homomorphism

$\lambda^{\prime}: \operatorname{Div}(X) \rightarrow(\mathbb{Z} / 2 \mathbb{Z})^{r}$

mapping a divisor $\mathfrak{a}$ to the $r$-tuple $\left(\varepsilon_{1}, \ldots, \varepsilon_{r}\right)$ with

$\varepsilon_{i}=\sum_{P \in \gamma_{i}} \operatorname{ord}_{P} \mathfrak{a} \bmod 2$.

Clearly $\lambda^{\prime}$ vanishes on $2 \operatorname{Div}(X)$. Now $\lambda^{\prime}$ also vanishes on $\mathfrak{H}(X)$ according to Theorem (3.4). Thus $\lambda^{\prime}$ induces a homomorphism

$\lambda: \operatorname{Pic}(X) / 2 \operatorname{Pic}(X) \rightarrow(\mathbb{Z} / 2 \mathbb{Z})^{r}$.

(5.1) Theorem. $\lambda$ is an isomorphism.

Proof. Since $\lambda$ is clearly surjective it suffices to show that $\operatorname{Pic}(X) / 2 \operatorname{Pic}(X)$ has cardinality $2^{r}$. We have a non-canonical decomposition

$\operatorname{Pic}(X) \cong \mathbb{Z} \times \operatorname{Pic}_{0}(X)$.

Thus it suffices to show that $\operatorname{Pic}_{0}(X) / 2 \operatorname{Pic}_{0}(X)$ has cardinality $2^{r-1}$. As explained in $\S 2$ (Lemma (2.7)) we identify $\operatorname{Pic}_{0}(X)$ with the group $J^{G}$ of $G$-invariant points of the Jacobian $J=\operatorname{Pic}_{0}(\bar{X})$. Now $2 \operatorname{Pic}_{0}(X)$ is contained in the subgroup $\mathrm{N}(J)$ of norms of $J$. On the other hand $J=2 J[\mathrm{Mu}, \mathrm{p} .42]$. Thus

$\mathrm{N}(J)=2 \mathrm{~N}(J) \subset 2 \operatorname{Pic}_{0}(X)$,

and we learn that $2 \operatorname{Pic}_{0}(X)$ coincides with $\mathrm{N}(J)$, hence

$\operatorname{Pic}_{0}(X) / 2 \operatorname{Pic}_{0}(X) \cong H^{0}(J)$.

Our theorem now follows from the fact observed in the preceding section that $H^{0}(J)$ has cardinality $2^{r-1}$.

Remark. Alling has given in the case $K=\mathbb{R}$ a description of the structure of $\operatorname{Pic}_{0}(X)$ using analytic techniques: As a real analytic subgroup of $J$ this group is isomorphic to $(\mathbb{R} / \mathbf{Z})^{8} \times(\mathbb{Z} / 2 \mathbb{Z})^{r-1}[\mathrm{Al}$, Th. 5.7].

The group $\operatorname{Pic}(X)$ can be regarded as the group of isomorphy classes $[\mathscr{L}]$ of invertible $\mathcal{O}_{X}$-modules $\mathscr{L}$ ( $\triangleq$ line bundles on $X$ ). We study the class $\left[\Omega_{X}\right]$ of the invertible $\mathcal{O}_{X}$-module $\Omega_{X}$ of germs of regular differentials on $X$ over $K$.

(5.2) Theorem. $\left[\Omega_{X}\right]$ lies in $2 \operatorname{Pic}(X)$.

Proof. Choose any rational differential $\omega \neq 0$ on $X$. Then $\left[\Omega_{X}\right]$ is the divisor class of the divisor of $\omega$. Thus $\lambda\left(\left[\Omega_{X}\right]\right)$ is the $r$-tuple $\left(\varepsilon_{1}, \ldots, \varepsilon_{r}\right)$ in $(\mathbb{Z} / 2 \mathbb{Z})^{r}$ with

$$
\varepsilon_{i} \equiv \sum_{P \in \gamma_{i}} \operatorname{ord}_{P}(\omega) \bmod 2 .
$$


Now

$$
\operatorname{ord}_{P}(\omega) \equiv \operatorname{res}_{P}\langle\omega\rangle \bmod 2
$$

for every $P$ in $\gamma$. Thus by the residue Theorem (3.4) all $\varepsilon_{i}$ are zero, hence $\left[\Omega_{X}\right]$ lies in $2 \operatorname{Pic}(X)$.

Remark. Theorem (5.2) does not remain true if $\gamma$ is empty. Indeed, we shall see in part II of this paper in the section about Witt rings of curves that in this case a divisor class $\alpha$ of $X$ lies in $2 \operatorname{Pic}(X)$ if and only if the degree of $\alpha$ is divisible by 4 . Thus $\left[\Omega_{X}\right]$ lies in $2 \operatorname{Pic}(X)$ if and only if the genus of $X$ is odd. For example, if $X$ is the projective quadric $x^{2}+y^{2}+z^{2}=0$ then $\left[\Omega_{X}\right]$ does not lie in $2 \operatorname{Pic}(X)$.

(5.3) Definition. A differential $\omega \neq 0$ in $\Omega_{F}$ is called definite if $\omega$ has even order at all real points $P$.

Theorem (5.2) means that there exist definite differentials on $X$. If two definite differentials $\omega$ and $\omega^{\prime}$ on $X$ are given then $\omega^{\prime}=f \omega$ with $f$ a real function definite on $\gamma$. We call $\omega$ and $\omega^{\prime}$ equivalent, if $f$ is positive definite on $\gamma$.

(5.4) Definition. An orientation of $\gamma$ is an equivalence class $[\omega]$ of definite differentials on $X$.

Clearly there exist $2^{r}$ orientations of $\gamma$. Furthermore by Lemma 2.14 every orientation of $\gamma$ can be repesented by a definite differential $\omega$ which has no zeros and no poles on $\gamma$.

Remark. In the case $K=\mathbb{R}$ this definition of orientation is compatible with the notion of orientation used in topology. Indeed, an orientation of $\gamma$ in the topological sense may well be regarded as a nowhere vanishing $C^{\infty}$-section of the $C^{\infty}$ tangent bundle of $\gamma$ modulo an obvious equivalence relation. Such a section corresponds to a nowhere vanishing $C^{\infty}$-section of the cotangent bundle of $\gamma$. Clearly every definite differential $\omega$ without zeros and poles on $\gamma$ yields such a section of the cotangent bundle of $\gamma$.

Since now we fix an orientation $\left[\omega_{0}\right]$ on $\gamma$ throughout the paper as long as we consider curves with $\gamma$ not empty. We define an additive map

$$
\partial_{P}: W(F) \rightarrow \mathbb{Z}
$$

for every $P$ in $\gamma$ by

$$
\partial_{P}(\varphi):=\operatorname{res}_{P}\left(\varphi \otimes\left\langle\omega_{0}\right\rangle\right) .
$$

These maps $\partial_{p}$ do not depend on the choice of the representative $\omega_{0}$ of our orientation, but only on the orientation itself. They are indeed, as is easily seen, maps as defined in (2.1) for an appropriate choice of the uniformizing parameters at the real points. According to our residue Theorem (3.3) we have

$$
\sum_{P \in \gamma_{i}} \partial_{P}(\varphi)=0
$$

for every form $\varphi$ over $F$ and every component $\gamma_{i}$. In the special case $K=\mathbb{R}$, $X$ the projective quadric $x^{2}+y^{2}-z^{2}=0$, this formula (5.6) has already been observed by Milnor and Husemoller [MH, p. 94]. 


\section{§6. Intervals}

We always assume that the set $\gamma$ of real points of our smooth complete curve $X$ is not empty, and we choose an orientation $\left[\omega_{0}\right]$ on $\gamma$ with corresponding maps $\partial_{P}: W(F) \rightarrow \mathbb{Z}$, as explained in the preceding section.

As before let $\gamma_{1}, \ldots, \gamma_{r}$ denote the components of $\gamma$. For every component $\gamma_{i}$ we choose once and for all a real function $f_{i} \neq 0$ which is negative definite on $\gamma_{i}$ and positive definite on the other components, cf. Theorem (2.10).

(6.1) Definition. Let $P$ and $Q$ be different real points on the same component $\gamma_{i}$. An interval function for the ordered pair $(P, Q)$ is a real function $\neq 0$ which is positive definite on $\gamma \backslash \gamma_{i}$, definite on $\gamma_{i} \backslash\{P, Q\}$, and fulfills $\partial_{P}(f)=-1$, hence according to our sum formula (5.6) $\partial_{Q}(f)=+1$.

Such a function exists for every pair $(P, Q)$ of different points on the same component $\gamma_{i}$. Indeed, according to our main theorem (4.5) we can find a real function $g \neq 0$ which has odd order on $\gamma$ precisely at $P$ and $Q$. Multiplying $g$ with some of the functions $f_{j}, j \neq i$, we arrive at a function $h$ which in addition is positive definite on $\gamma \backslash \gamma_{i}$. Now put $f=f_{i} h$ if $\partial_{P}(h)=+1$, and put $f=h$ in the case $\partial_{P}(h)=-1$. We have $\partial_{P}(f)=-1$, hence $f$ is an interval function for $(P, Q)$.

(6.2) Definition. Let $P$ and $Q$ again be different real points on the same component $\gamma_{i}$, and let $f$ be an interval function for $(P, Q)$. The open interval $] P, Q[$ is defined as the set of all points $R$ in $\gamma$ with $\tau_{R}(f)=-1$.

Clearly $] P, Q\left[\right.$ is contained in $\gamma_{i}$. This set does not depend on the choice of the interval function $f$, since any other interval function for $(P, Q)$ arises from $f$ by multiplication with a real function positive definite on $\gamma$. The set $] P, Q[$ must be infinite, since, otherwise $f$ would be positive definite on $\gamma$ except at finitely many points and thus positive definite everywhere on $\gamma$ (cf. Theorem 2.3). Furthermore $] P, Q\left[\right.$ is indeed an open subset of $\gamma_{i}$ : For $R$ in $] P, Q[$ we can find a real function $g \neq 0$ such that $h:=f g^{2}$ has no pole or zero in $R$. The set $h^{-1}(] \infty, 0[)$ is an open neighborhood of $R$ contained in $] P, Q[$.

The reader will easily verify that if $X$ is the projective line over $K$ we obtain by our definition 6.2 the same open intervals on $K \cup \infty$ as defined in the introduction, $\S 1$, by choosing as interval functions products of $\leqq 2$ linear polynomials.

(6.3) Proposition. Let $(P, Q)$ be a pair of different points on the same component of $\gamma$, and let $\left(P^{\prime}, Q^{\prime}\right)$ be a second such pair. Assume $] P, Q[=] P^{\prime}, Q^{\prime}\left[\right.$. Then $P=P^{\prime}$ and $Q=Q^{\prime}$.

Proof. Let $f$ be an interval function for $(P, Q)$ and $f^{\prime}$ an interval function for $\left(P^{\prime}, Q^{\prime}\right)$. Then $f f^{\prime}$ is positive definite on $\gamma$ except possibly at $P, Q, P^{\prime}$, and $Q^{\prime}$. Thus $f f^{\prime}$ is positive definite everywhere on $\gamma$, and $\partial_{R}(f)=\partial_{R}\left(f^{\prime}\right)$ for all $R$ in $\gamma$. This implies $P=P^{\prime}$ and $Q=Q^{\prime}$.

Thus for an open interval $I$ we have a unique point $P$ and a unique point $Q$ such that $I=] P, Q[$. Justified by this fact we often call an interval function for $(P, Q)$ an "interval function for $I$," and we call $P$ and $Q$ the "endpoints" of $I$.

We want to verify for our intervals geometric properties which are analogous to evident properties of the classical open intervals on the circle $S^{1}$. Without loss of generality we confine our interest to intervals on $\gamma_{1}$. 
(6.4) Proposition. Let $P$ and $Q$ be different points on $\gamma_{1}$. Then $\gamma_{1}$ is the disjoint union of the sets $] P, Q[] Q,, P[,\{P\}$, and $\{Q\}$.

Proof. If $f$ is an interval function for $(P, Q)$, then

$$
g:=-f f_{2} \ldots f_{r}
$$

is an interval function for $(Q, P)$. Indeed, $g$ is positive definite on $\gamma \backslash \gamma_{1}$, definite on $\gamma_{1} \backslash\{P, Q\}$, and fulfills $\partial_{P}(g)=+1, \partial_{Q}(g)=-1$. The points $R$ of $\gamma_{1}$ not in $] P, Q[$ and different from $P$ and $Q$ are precisely all points with $\tau_{R}(g)=-1$.

For $P$ and $Q$ points on the same component, say $\gamma_{1}$, we define the closed interval $[P, Q]$ as the union of $] P, Q[$ with the endpoints $P$ and $Q$. According to Proposition 6.4 just proved $[P, Q]$ is the complement of $] Q, P\left[\right.$ in $\gamma_{1}$. Thus $[P, Q]$ is indeed a closed subset of $\gamma_{1}$. In $\S 7$ we shall see that $[P, Q]$ is actually the closure of $] P, Q[$ in $\gamma$.

We return to open intervals.

(6.5) Proposition. Let $R$ be a point in the open interval $] P, Q[$. Then $] P, Q[$ is the disjoint union of the sets $] P, R[] R,, Q[$ and $\{R\}$.

Proof. We assume that $P$ and $Q$ lie on $\gamma_{1}$. Let $f$ be an interval function for $] P, Q[$ and $g$ an interval function for ]P, $R$ [. According to Lemma 2.11 there exists a real function $u \neq 0$ such that

(*) $\langle 1,-f\rangle \otimes\langle 1,-g\rangle \cong 2 \times\langle 1,-u\rangle$.

Let us look at $u$ more closely. Applying $\partial_{T}$ to (*) for $T$ a real point different from $P, Q, R$ we learn that $\partial_{T}(u)=0$, i.e. $u$ is definite at $T$. Applying $\tau_{T}$ to (*) for $T$ in $\gamma \backslash \gamma_{1}$ we see that $u$ is positive definite on $\gamma \backslash \gamma_{1}$. Applying $\partial_{P}$ and $\partial_{R}$ we get

$$
\begin{aligned}
& -2 \partial_{P}(u)=-\partial_{P}(f)-\partial_{P}(g)=+2, \\
& -2 \partial_{R}(u)=-\left(1-\tau_{R}(f)\right) \partial_{R}(g)=-2 .
\end{aligned}
$$

Thus $\partial_{P}(u)=-1$ and $\partial_{R}(u)=+1$. From our sum formula (5.6) we finally get $\partial_{Q}(u)=0$. Thus $u$ is an interval function for $] P, R[$, and $u g$ is positive definite on $\gamma$. By Theorem (4.1) $u g$ is a sum of two squares in $F$ and our relation (*) simplifies to

$(* *)\langle 1,-f\rangle \otimes\langle 1,-g\rangle \cong 2 \times\langle 1,-g\rangle$.

Applying $\tau_{T}$ to (**) for $T$ a real point different from $P$ and $R$, we learn

]$P, Q[\cap] P, R[=] P, R[$.

\{Consider separately the cases $T \neq Q$ and $T=Q$.$\} Thus ] P, R[$ is contained in ]$P, Q[$.

The function $h:=f g$ is an interval function for $] R, Q[$. Indeed, $h$ is positive definite on $\gamma \backslash \gamma_{1}$, definite on $\gamma_{1}$ except in $R$ and $Q$, and

$$
\partial_{R}(h)=\tau_{R}(f) \partial_{R}(g)=-1 .
$$

Multiplying the obvious relation

$$
\langle 1,-h\rangle \sim\langle 1,-f\rangle \perp f\langle 1,-g\rangle
$$


with the form $\langle 1,-f\rangle$ and using $(* *)$ we obtain

$$
\langle 1,-f\rangle \otimes\langle 1,-h\rangle \cong 2 \times\langle 1,-h\rangle \text {. }
$$

From this relation we obtain as above that $] R, Q[$ is contained in $] P, Q[$.

For $S$ a point on $\gamma_{1}$ different from $P, Q$, and $R$ we have

$$
\tau_{s}(f) \tau_{s}(g) \tau_{s}(h)=1 .
$$

From this equation we learn that for every point $S$ in $] P, Q[$ different from $R$ precisely one of the signs $\tau_{s}(g), \tau_{s}(h)$ is -1 . This tells us that $] P, Q[$ is indeed the disjoint union of $] P, R[] R,, Q[$, and $\{R\}$. q.e.d.

We now choose a point $P_{0}$ on $\gamma_{1}$ and introduce on $\gamma_{1}^{\prime}:=\gamma_{1} \backslash\left\{P_{0}\right\}$ an ordering by the following convention: If $P$ and $Q$ are points in $\gamma_{1}^{\prime}$ then $P \leqq Q$ if and only if the interval $] P_{0}, P[$ is contained in $] P_{0}, Q[$. The rule

$$
P \leqq Q, \quad Q \leqq R \Rightarrow P \leqq R
$$

is evident, and the rule

$$
P \leqq Q, \quad Q \leqq P \Rightarrow P=Q
$$

follows from Proposition 6.3. Thus we have indeed defined an ordering on $\gamma_{1}^{\prime}$.

(6.6) Proposition. i) $\gamma_{1}^{\prime}$ is totally ordered.

ii) For every $Q$ in $\gamma_{1}^{\prime}$ the interval $] P_{0}, Q\left[\right.$ is the set of all $R$ in $\gamma_{1}^{\prime}$ with $R<Q$, and ]$Q, P_{0}\left[\right.$ is the set of all $R$ in $\gamma_{1}^{\prime}$ with $R>Q$.

iii) If $P$ and $Q$ are points in $\gamma_{1}^{\prime}$ with $P<Q$ then $] P, Q\left[\right.$ is the set of all $R$ in $\gamma_{1}^{\prime}$ with $P<R<Q$.

Proof. a) Let $P$ and $Q$ be different points in $\gamma_{1}^{\prime}$. If $P$ lies in $] P_{0}, Q[$ then $] P_{0}, P[$ is contained in $] P_{0}, Q[$ according to the preceding Proposition 6.5, hence $P<Q$. Assume now that $P$ does not lie in $] P_{0}, Q[$. Then $P$ must lie in $] Q, P_{0}[$ by Proposition (6.4). Again by Proposition (6.5) $] P, P_{0}[$ is contained in $] Q, P_{0}[$. Taking complements in $\gamma_{1}$ we get $\left[P_{0}, Q\right] \subset\left[P_{0}, P\right]$, and a fortiori $] P_{0}, Q\left[\subset\left[P_{0}, P\right]\right.$. Since the points $P_{0}$ and $P$ do not lie in $] P_{0}, Q[$, we have $] P_{0}, Q[\subset] P_{0}, P[$, i.e. $Q<P$. This discussion shows that $\gamma_{1}^{\prime}$ is totally ordered, that $] P_{0}, Q\left[\right.$ is the set of all $P$ in $\gamma_{1}^{\prime}$ with $P<Q$, and that $] Q, P_{0}$ [ is the set of all $P$ in $\gamma_{1}^{\prime}$ with $P>Q$.

b) It remains to prove the third assertion (iii). Let $P$ and $Q$ be points in $\gamma_{1}^{\prime}$ with $P<Q$. The interval $] P_{0}, Q[$ is the disjoint union of $] P_{0}, P[] P,, Q[$ and $\{P\}$. As already shown $] P_{0}, P\left[\right.$ consists of the points $R$ of $\gamma_{1}^{\prime}$ with $R<P$, and $] P_{0}, Q[$ consists of the points $R$ with $R<Q$. Thus ] $P, Q\left[\right.$ consists of the points $R$ in $\gamma_{1}^{\prime}$ with $P<R<Q$. q.e.d.

From this proposition we immediately obtain

(6.7) Corollary. Let $P_{1}<P_{2}<\cdots<P_{t}$ be finitely many points in $\gamma_{1} \backslash\left\{P_{0}\right\}$. Then $\gamma_{1}$ is the disjoint union of the finite set $\left\{P_{0}, P_{1}, \ldots, P_{t}\right\}$ and the intervals $] P_{i}, P_{i+1}[$, $0 \leqq i \leqq t-1$, and $] P_{t}, P_{0}[$.

We can now make the sign behavior of a real function more explicit. 
(6.8) Theorem. Let $u \neq 0$ be a real function which is not definite on $\gamma_{1}$, and let $P_{0}, \ldots, P_{2 s-1}$ be the points of odd order of $u$ on $\gamma_{1}(s \geqq 1)$. Assume $\partial_{P_{0}}(u)=-1$, and with respect to $P_{0}$

$$
P_{1}<P_{2}<\cdots<P_{2 s-1} \text {. }
$$

Then for $0 \leqq i \leqq 2 s-1$

$$
\left.\partial_{P_{i}}(u)=-1\right)^{i+1} \text {. }
$$

Furthermore for $P$ a point on $\gamma_{1}$ different from the $P_{i}$ the sign $\tau_{P}(u)$ is -1 if and only if $P$ lies in one of the disjoint intervals $] P_{2 i}, P_{2 i+1}[, 0 \leqq i \leqq s-1$.

Thus if $u$ has odd order at a real point $P$ we may say that " $u$ changes sign at $P$ from -1 to +1 " if $\partial_{P}(u)=+1$, and that " $u$ changes sign at $P$ from +1 to $-1 "$ if $\partial_{P}(u)=-1$.

For the proof of Theorem (6.8) we choose interval functions $g_{i}$ for the $s$ intervals $] P_{2 i}, P_{2 i+1}[, 0 \leqq i \leqq s-1$, and put

$$
v:=u g_{0} \ldots g_{s-1} \text {. }
$$

Since $g_{i}$ has odd order precisely at $P_{2 i}$ and $P_{2 i+1}$ this function $v$ is definite on $\gamma_{1}$. The point $P_{0}$ does not lie in any of the intervals $] P_{2 i}, P_{2 i+1}$ [ with $1 \leqq i \leqq s-1$. Thus $\tau_{P_{0}}\left(g_{i}\right)=+1$ for $1 \leqq i \leqq s-1$. Furthermore $\partial_{P_{0}}(u)$ and $\partial_{P_{0}}\left(g_{0}\right)$ are both -1 . Thus we see that $\tau_{P_{0}}(v)=+1$, hence $v$ is positive definite on $\gamma_{1}$. This implies that $u$ and $g_{0} \ldots g_{s-1}$ have the same value under $\tau_{P}$ and also under $\partial_{P}$ for every $P$ in $\gamma_{1}$. The statements of Theorem 6.8 now follow from the preceding Corollary (6.7).

We state an obvious consequence of Theorem (6.8).

(6.9) Corollary. Let $] P, Q[$ be an open interval on $\gamma$, and let $u \neq 0$ be a real function without points of odd order in $] P, Q[$. Then for all $R$ in $] P, Q\left[\right.$ the sign $\tau_{R}(u)$ is the same.

As an application of our theory of intervals we determine the components of the set $\beta$ of real points of a Zariski open subset $Z$ of $X$, as defined in $\S 2$.

(6.10) Theorem. Every component of $\beta$ is contained in a component $\gamma_{i}$ of $\gamma$. Let $S_{1}$ denote the finite set of points in $\gamma_{1}$ not contained in $\beta$, and $t$ the cardinality of $S_{1}$. If $t \leqq 1$ then $\gamma_{1} \backslash S_{1}$ is a component of $\beta$. Assume now $t \geqq 2$ and let $P_{0}, \ldots, P_{t-1}$ denote the points of $S_{1}$. In the case $t \geqq 3$ assume further without loss of generality

$$
P_{1}<P_{2}<\cdots<P_{t-1}
$$

with respect to $P_{0}$. The components of $\beta$ contained in $\gamma_{1}$ are precisely the $t$ intervals ]$P_{i}, P_{i+1}[, 0 \leqq i \leqq t-2$, and $] P_{t-1}, P_{0}[$.

Proof. a) The first assertion is trivial. If $t \leqq 1$ then every real function $u$ definite on $\gamma_{1} \backslash S_{1}$ must be definite on $\gamma_{1}$, since the number of points of odd order for $u$ on $\gamma_{1}$ is even. Thus $\gamma_{1} \backslash S_{1}$ is a component of $\beta$.

b) Assume now $t \geqq 2$, and let $\mathscr{F}$ denote the family of open intervals consisting of $] P_{i}, P_{i+1}[$ for $0 \leqq i \leqq t-2$ and of $] P_{t-1}, P_{0}$ [. Every function definite on $\beta$ has constant sign on every interval in $\mathscr{F}$ by the preceding Corollary 6.9. Now let $P$ 
and $Q$ be points of $\gamma_{1} \backslash S_{1}$ lying in different intervals of the family $\mathscr{F}$. Let $] R, S[$ be the interval in $\mathscr{F}$ containing $P$, and let $u$ denote an interval function for $] R, S[$. Then $u$ is definite on $\beta$ and $u$ has opposite sign at $P$ and $Q$. This discussion shows that indeed $\mathscr{F}$ is the set of components of $\beta$ contained in $\gamma_{1}$.

\section{Appendix A : A Remark on the Topology of the Set of Real Points}

Our absolute theory completely disregards the fact that in practice most often real curves come up as subschemes of other algebraic schemes over $K$. This urges me to add a remark on the topology of the set $Z(K)$ of real points of a smooth irreducible curve $Z$ over $K$.

For every algebraic scheme $Y$ over $K$ a topology on the set $Y(K)$ of real points ( = closed points with residue class field $K$ ) can be defined as follows [S, p. $309 \mathrm{ff}$ ]: Let $U$ be a Zariski open subset of $Y$, further $f_{1}, \ldots, f_{m}$ regular functions on $U$, and finally $\varepsilon$ a positive constant in $K$. We introduce the set $V\left(U ; f_{1}, \ldots, f_{m} ; \varepsilon\right)$ consisting of all $x$ in $U(K)$ with

$$
-\varepsilon<f_{i}(x)<\varepsilon \quad \text { for } 1 \leqq i \leqq m .
$$

The "strong topology" on $Y(K)$ is defined as the topology with these sets $V(U$; $\left.f_{1}, \ldots, f_{m} ; \varepsilon\right)$ as basis.

If $Y^{\prime}$ is a Zariski locally closed (open, closed) subscheme of $Y$, then clearly $Y^{\prime}(K)$ is a locally closed (open, closed) subset of $Y(K)$ and the strong topology of $Y(K)$ induces on the subset $Y^{\prime}(K)$ again the strong topology. In the special case that $Y$ is the affine standard space $\mathbb{A}^{n}$ over $K$ the strong topology on $Y(K)=K^{n}$ coincides with the product topology coming from the interval topology on $K$, as is easily shown.

Now let $Z$ be a smooth irreducible curve over $K$. We may regard $Z$ as a Zariski open subset of a complete smooth irreducible curve $X$. We realize that the topology on $X(K)$ introduced in $\S 1$ coincides with the strong topology, and thus the topology of $\S 1$ induces on $Z(K)$ again the strong topology. Now the following proposition is obvious:

Proposition. Assume the smooth irreducible curve $Z$ is a locally closed subscheme of $\mathbb{A}^{n}$ for some $n \geqq 1$. Then $Z(K)$ is locally closed in $K^{n}$ with respect to the product topology, and $K^{n}$ induces on $Z(K)$ the topology introduced in $\S 1$.

\section{Appendix B : Residues of Diferentials}

Assume that $\gamma$ is not empty and, as before, let $\gamma_{1}, \ldots, \gamma_{r}$ denote the components of $\gamma$. In $\S 3$ we have seen that for $\varphi$ a dfferential valued form over $F$ the sum of residues of $\varphi$ on each component $\gamma_{i}$ is zexo. The question arises whether the analogous statement holds for the pesidues ondifferentials themselves. This is indeed true.

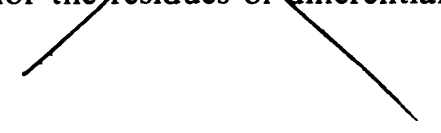


Theorem. For every differential $\omega$ in $\Omega_{F}$ and every component $\gamma_{i}$

$$
\sum_{P \in \gamma_{i}} \operatorname{res}_{P}(\omega)=0
$$

Proof. We verify this formula for say, $\gamma_{1}$. We may assume $\omega \neq 0$. Let $\mathfrak{p}_{1}, \ldots, \mathfrak{p}_{s}$ denote the real and complex points putside $\gamma_{1}$ where $\omega$ has odd order. From the residue formula (3.3), applied to the form $\langle\omega\rangle$, we learn that in case $r \geqq 2$ there lies on each component $\gamma_{i}$ with $2 \leqq i \leqq r$ gn even number of the points $\mathfrak{p}_{1}, \ldots, \mathfrak{p}_{s}$. Thus according to Theorem (5.1) the class of the divisor $\mathfrak{p}_{1} \ldots \mathfrak{p}_{s}$ in $\operatorname{Pic}(X)$ lies in $2 \mathrm{Pic}(X)$. This means that there exists a reanction $g \neq 0$ such that

$$
\operatorname{div}(g)=\mathfrak{p}_{1} \ldots \mathfrak{p}_{2} \mathfrak{a}^{2}
$$

with a some divisor on $X$. The function $g$ is clearly defpite on $\gamma_{1}$. Multiplying $g$ by an appropriate real function definite on $\gamma$ we may assume that $g$ is positive definite on $\gamma_{1}$, cf. Theorem (2.10). Then using the weak approximation theorem for valuations we fipd a real function $h$ such that $\left(g h^{2}\right)(P)=1$ for alt the finitely many points $P$ on $\gamma_{/}$where $\omega$ has odd order. Now applying the classical kesidue formula $\left[\mathrm{Lg}_{1}, \mathrm{p} .248\right]$ to the differential $g h^{2} \omega$ on $\bar{X}$ we obtain

$$
0=\sum_{p \in X} \operatorname{Tr}_{K(\mathfrak{p}) / K} \operatorname{res}_{\mathfrak{p}}\left(g h^{2} \omega\right)=\sum_{P \in \gamma_{1}} \operatorname{res}_{P}(\omega) \text {. q.e.d. }
$$

\section{References}

Ar. Arason, J.K.: Cohomologische Invarianten quadratischer Formen. J. Algebra 36, 448-491 (1975)

A. Artin, E.: Über die Zerlegung definiter Funktionen in Quadrate, Abh. math. Sem. Univ. Hamburg 5, 100-115 (1927)

Al. Alling, N. L.: Analytic geometry on real algebraic curves. Math. Ann. 207, 23-46 (1974)

EL. Elman, R., Lam, T.Y.: Quadratic forms under algebraic extensions. Math. Ann. 219, 21-42 (1976)

G. Geyer, W.D.: Dualität bei abelschen Varietäten über reell abgeschlossenen Körpern. J. reine angew. Math. to appear

$G_{1}$. Geyer, W.D.: Ein algebraischer Beweis des Satzes von Weichold über reelle algebraische Funktionenkörper. In: Algebraische Zahlentheorie (Oberwolfach 1964), pp. 83-98. Mannheim: Bibliographisches Institut 1967

GHKS. Geyer, W.D., Harder, G., Knebusch, M., Scharlau, W.: Ein Residuensatz für symmetrische Bilinearformen, Inventiones math. 11, 319-328 (1970)

K. Knebusch, M.: Grothendieck- und Wittringe von nichtausgearteten symmetrischen Bilinearformen. S.-ber. Heidelberger Akad. Wiss. math.-naturw. Kl. 1969/70, 3. Abhdl. 96 pp. (1970)

$\mathrm{K}_{1} \quad$ Knebusch, M.: On the uniqueness of real closures and the existence of real places, Commentarii math. Helvet. 47, 260-269 (1972)

L. Lam, T.Y.: The algebraic theory of quadratic forms. Reading: Benjamin 1973

Lg. Lang, S.: The theory of real places, Ann. of Math. II. Ser. 57, 378-391 (1953)

$\mathrm{Lg}_{1} \quad$ Lang, S.: Introduction to algebraic geometry. New York: Interscience 1958

M. Milnor, J.: Topology from the differentiable viewpoint. Charlottesville University Press of Virginia, 1965

MH. Milnor, J., Husemoller, D.: Symmetric bilinear forms, Berlin-Heidelberg-New York: Springer 1973 
Mu. Mumford, D.: Abelian Varieties. Oxford: University Press 1970

P. Pfister, A.: Zur Darstellung definiter Funktionen als Summen von Quadraten, Inventiones math. 4, 229-237 (1967)

S. Shafarevich, I. R.: Basic algebraic geometry, Berlin-Heidelberg-New York: Springer 1974

T. Tate, J.: WC-groups over p-adic fields, In: Séminaire Bourbaki 10e année 1957/58, exposé no. 156,13 pp. Paris: Secrétariat mathématique 1958

W. Witt, E.: Zerlegung reeller algebraischer Funktionen in Quadrate, Schiefkörper über reellem Funktionenkörper. J. reine angew. Math. 171, 4-11 (1934)

Received March 2, 1976 


\section{Berichtigung zu der Arbeit: On Algebraic Curves over Real Closed Fields}

Math. Z. 150, $49-70$ (1976)

Manfred Knebusch

Fachbereich Mathematik der Universität, D-8400 Regensburg, Bundesrepublik Deutschland

In Appendix B der Arbeit wurde der Satz aufgestellt, da $B$ für jedes Differential $\omega$ der vollständigen glatten Kurve $X$ über einen reell abgeschlossenen Körper $k$ die Summe der Residuen in den Punkten einer Komponente $\gamma_{i}$ der Menge der reellen Punkte von $X$ verschwindet. Dieser Satz ist falsch, wie etwa das Differential $\omega=\left(1+x^{2}\right)^{-1} x d x$ auf der projektiven Geraden zeigt, mit $x$ eine Erzeugende des Funktionenkörpers. Der Fehler im „Beweis“ des Satzes beruht auf einer fehlerhaften Anwendung des klassischen Residuensatzes in der letzten Zeile der Arbeit.

Ich wurde auf diesen Fehler von Herrn W.D. Geyer aufmerksam gemacht. Der Fehler berührt die anderen Resultate der Arbeit nicht, auch nicht die von Teil II (Math. Z. 151, 1976), weil Appendix B nirgends benutzt wird. 